\title{
REMAINDERS IN HAUSDORFF COMPACTIFICATIONS
}

\author{
RICHARD E. CHANDLER AND FU-CHIEN TZUNG
}

\begin{abstract}
We generalize a theorem of Steiner and Steiner and use it to obtain new results concerning remainders for completely regular spaces. Both the locally compact and the nonlocally compact cases are considered.
\end{abstract}

Introduction. The nature of what one added to a completely regular space in order to compactify it has been considered almost from the beginning of the theory. For example, Čech [2] was concerned about the cardinality of $\beta \mathbf{N} \backslash \mathbf{N}$. It was not until the mid-1960's, however, that the topological properties of remainders was studied in a concerted fashion. Magill, in a series of papers [8], [9], [10] discovered and developed conditions on a space $X$ which would guarantee that members of a certain class of continua would be remainders of $X$. This theory was extended by Rogers [14] and Chandler $[3,7.8]$ to conclude that if $X$ is locally compact and not pseudocompact then any compact space containing a dense continuous image of $\mathbf{R}$ is a remainder of $X$. One of the useful results in this area has been a theorem of Steiner and Steiner [15]. Here we generalize this theorem and use it to obtain some new results on remainders. Throughout we follow the general terminology of [3]. In particular, all spaces will be assumed to be completely regular and Hausdorff. A remainder of $X$ is any $\alpha X \backslash X$ where $\alpha X$ is a compactification of $X$.

1. The locally compact case. Throughout this section we assume all spaces to be locally compact. If $f: X \rightarrow Y$ is continuous and $Y$ is compact then $\mathcal{L}\{f\}$ will denote the set $\cap\left\{\operatorname{cl}_{Y} f(X \backslash F) \mid F \subseteq X\right.$ is compact $\}$.

THEOREM 1. $\mathcal{L}\{f\}$ is a remainder of $X$.

Proof. First, we show that if $X$ is not compact then $\mathcal{L}\{f\}$ is not empty. We do this by showing $\left\{\mathrm{cl}_{Y} f(X \backslash F) \mid F \subseteq X\right.$ is compact $\}$ is a family of closed subsets of $Y$ which has the finite intersection property. For suppose not. Then there is a family $\left\{F_{1}, \ldots, F_{n}\right\}$ of compact subsets of $X$ for which $\operatorname{cl}_{Y} f(X \backslash$ $\left.F_{1}\right) \cap \cdots \cap \operatorname{cl}_{Y} f\left(X \backslash F_{n}\right)=\varnothing$. Thus $Y=G_{1} \cup \cdots \cup G_{n}$ where $G_{i}=Y \backslash$ $\operatorname{cl}_{Y} f\left(X \backslash F_{i}\right)$. Then $X=f^{-1}\left(G_{1}\right) \cup \cdots \cup f^{-1}\left(G_{n}\right)$. Now $G_{i} \subseteq Y \backslash f\left(X \backslash F_{i}\right)$ so that $f^{-1}\left(G_{i}\right) \subseteq f^{-1}\left(Y \backslash f\left(X \backslash F_{i}\right)\right) \subseteq F_{i}$. We conclude that $X=F_{1}$

Received by the editors August 22, 1977.

AMS (MOS) subject classifications (1970). Primary 54D40.

(c) American Mathematical Society 1978 
$\cup \cdots \cup F_{n}$, which is compact. This contradiction assures us that $\mathcal{E}\{f\}$ $\neq \varnothing$.

Let $f^{\beta}: \beta X \rightarrow Y$ extend $f$. We claim that $f^{\beta}(\beta X \backslash X)=\mathcal{L}\{f\}$. Since $f^{\beta}(\beta X \backslash X)$ is compact, all we need to show is that $f^{\beta}(\beta X \backslash X)$ is contained in and dense in $\mathcal{L}\{f\}$. For any compact $F \subseteq X$ we have $\operatorname{cl}_{\beta X}(X \backslash F) \cap(\beta X$ $\backslash X)=\beta X \backslash X$ so that $f^{\beta}(\beta X \backslash X) \subseteq \operatorname{cl}_{Y} f(X \backslash F)$. Since $F$ is arbitrary, we conclude that $f^{\beta}(\beta X \backslash X) \subseteq \mathcal{L}\{f\}$. If $f^{\beta}(\beta X \backslash X)$ is not dense in $\mathcal{L}\{f\}$ then there is a point $p \in \mathcal{L}\{f\}$ and a compact neighborhood $U$ of $p$ such that $U \cap f^{\beta}(\beta X \backslash X)=\varnothing$. Then $G=\left(f^{\beta}\right)^{-1}(U) \subseteq X \subseteq \beta X$, and since $U$ is closed in $Y$ we have $G$ is compact. $p \notin \operatorname{cl}_{Y} f(X \backslash G)$, contradicting $p \in \mathcal{L}\{f\}$ $\subseteq \operatorname{cl}_{Y} f(X \backslash G)$.

We have now shown that $\mathcal{L}\{f\}$ is a continuous image of $\beta X \backslash X$. By a theorem of Magill [8] it follows that $\mathcal{L}\{f\}$ is a remainder of $X$.

Corollary 1. Any of the following implies that $\mathcal{L}\{f\}=Y$.

(1) $f(X \backslash F)$ is dense in $Y$ whenever $F \subseteq X$ is compact.

(2) $f^{-1}(Z)$ is not compact for any zero set $Z \subseteq Y$.

(3) $f^{-1}(p)$ is not compact for each $p \in D$, a dense subset of $Y$.

Proof. That (1) implies $\mathcal{L}\{f\}=Y$ is obvious. The hypothesis of (2) implies $f(X \backslash F) \cap Z \neq \varnothing$ for any compact $F \subseteq X$ and any zero set $Z \subseteq Y$. Since any point in $Y$ has a base of zero set neighborhoods, it follows that $f(X \backslash F)$ is dense in $Y$ for each compact $F \subseteq X$. Similarly the hypothesis of (3) implies that $f^{-1}(p)$ is contained in no compact $F \subseteq X$. Thus $D \subseteq f(X \backslash$ $F$ ) and we conclude that $f(X \backslash F)$ is dense in $Y$ for each compact $F \subseteq X$.

The theorem that (1) implies $\mathcal{L}\{f\}=Y$ is the result of Steiner and Steiner referred to in the Introduction. That (3) implies $\mathcal{L}\{f\}=Y$ is due to Magill [8]. We can use this corollary to obtain an old remainder theorem originally due to Alexandroff and Urysohn [1] (although it was stated somewhat differently) and improved by Engelking [5].

COROllary 2. Let $D$ be an infinite discrete space and suppose $Y$ is compact with density character less than or equal to $|D|$. Then $Y$ is a remainder of $D$.

Proof. The only compact subsets of $D$ are finite. Thus, if $f$ maps $D$ onto a dense subset of $Y$ we have $f(D \backslash F)$ is dense in $Y$ whenever $F$ is compact in $X$. (For any isolated point $p$ of $Y$, let $f$ send infinitely many points of $D$ to $p$.)

Since there are separable compact spaces with cardinality $2^{c}$, we conclude that there are remainders of $\mathbf{N}$ with this cardinality. Thus $|\beta \mathbf{N} \backslash \mathbf{N}| \geqslant 2^{c}$. Cech had observed that $|\beta \mathbf{N} \backslash \mathbf{N}| \leqslant 2^{c}$. Thus $|\beta \mathbf{N} \backslash \mathbf{N}|=2^{c}$, a result originally due to Pospišil [12]. Mrórwka [11] apparently was the first to discover this technique of proof.

The result of Steiner and Steiner above cannot be used to produce disconnected remainders for a connected space $X$. Theorem 1 can do this: Let $X=(0,1), Y=[0,1]$, and $f: X \rightarrow Y$ is the inclusion map. Then $\mathcal{E}\{f\}=$ $\{0,1\}$. 
We can also use Theorem 1 to give a necessary and sufficient condition for a space to have a remainder consisting of exactly $n$ points. The first to characterize such spaces was Magill [7]. We use his result to obtain ours. Let $T_{n}$ denote all points in $\mathbf{R}^{n}$ on the positive coordinate axes whose distance to the origin is less than or equal to $1 .\left(T_{n}\right.$ is the " $n$-ode", the obvious generalization of the triode.) Let $\left\{e_{1}, \ldots, e_{n}\right\}$ denote the set of endpoints in $T_{n}$, namely those points with one coordinate equal to 1 and all the others zero.

THEOREM 2. $X$ has an n-point remainder if and only if there is a map $f$ : $X \rightarrow T_{n}$ with $\mathcal{L}\{f\}=\left\{e_{1}, \ldots, e_{n}\right\}$.

Proof. The "if" part is immediate from Theorem 1. Conversely, suppose $X$ has a compactification with an $n$-point remainder. By Magill's theorem referred to above, it is possible to write $X=K \cup G_{1} \cup \cdots \cup G_{n}$, where $K$ is compact, $G_{i}$ is open, $G_{i} \cup K$ is not compact, $G_{i} \cap K=\varnothing, G_{i} \cap G_{j}=\varnothing$ if $i \neq j$, for $i=1,2, \ldots, n$. In the compactification $\alpha X$ which Magill constructs using this representation of $X$ we have each $G_{i}$ associated with a point $p_{i}$ in $\alpha X \backslash X$. Let $g_{i}: \alpha X \rightarrow[0,1]$ be defined in such a way that $g_{i}\left(p_{i}\right)=1$ and $g_{i}\left(\alpha X \backslash\left[G_{i} \cup\left\{p_{i}\right\}\right]\right)=0$. Define $\hat{f}: X \rightarrow T_{n}$ by letting the $i$ th coordinate of $\hat{f}(t)$ be equal to $g_{i}(t)$. Since for a given point $t \in \alpha X$ at most one $g_{i}$ is different from zero at $t$, it is clear that $\hat{f}(t) \in T_{n}$.

If $f=\left.\hat{f}\right|_{X}$ then $\left\{e_{1}, \ldots, e_{n}\right\} \subseteq \mathcal{L}\{f\}$. For any point $t \in T_{n}$ with $t \neq e_{i}$, $i=1,2, \ldots, n$, we can choose a compact neighborhood $U$ of $t$ in $T_{n}$ with no $e_{i}$ in $U . f^{-1}(U)=\hat{f}^{-1}(U)$ which is compact. $t \notin \mathrm{cl} f\left(X \backslash f^{-1}(U)\right)$ so that $t \notin \mathcal{L}\{f\}$. Therefore $\mathcal{L}\{f\}=\left\{e_{1}, \ldots, e_{n}\right\}$.

Corollary 1. Suppose $\alpha X \backslash X=\left\{p_{1}, \ldots, p_{n}\right\}$ and each $\left\{p_{i}\right\}$ is a zero set in $\alpha X$. Then any remainder of $T_{n}^{0}=T_{n} \backslash\left\{e_{1}, \ldots, e_{n}\right\}$ is a remainder of $X$.

Proof. For each $i$, there is a map $h_{i}: \alpha X \rightarrow[0,1]$ with $h_{i}\left(p_{i}\right)=1$ and $h_{i}(t)<1$, if $t \neq p_{i}$. Let $f_{i}: \alpha X \rightarrow[0,1]$ be defined by $f_{i}(t)=h_{i}(t) g_{i}(t)$ where the $g_{i}$ are as described in the proof of Theorem 2. Then $f_{i}: \alpha X \rightarrow[0,1]$ with $f_{i}\left(p_{i}\right)=1, f_{i}(t)<1$ if $t \neq p_{i}$, and $f_{i}(t)=0$ if $t \notin G_{i}$. Define $f: \alpha X \rightarrow T_{n}$ by $f(t)_{i}=f_{i}(t)$. Then $f: \alpha X \rightarrow T_{n}$ with $f\left(p_{i}\right)=e_{i}$ and $f(t) \in T_{n}^{0}$ if $t \neq e_{i}, i=$ $1,2, \ldots, n$.

Suppose $\gamma T_{n}^{0}$ is any compactification of $T_{n}^{0}$ where we consider $\gamma: T_{n}^{0} \rightarrow \gamma T_{n}^{0}$ as the inclusion map. It is clear that

$$
\gamma \circ\left(\left.f\right|_{X}\right): X \rightarrow \gamma T_{n}^{0} \text { and } \mathcal{L}\left\{\gamma \circ\left(\left.f\right|_{X}\right)\right\}=\gamma T_{n}^{0} \backslash T_{n}^{0} .
$$

Note that the requirement that each $\left\{p_{i}\right\}$ be a zero set in $\alpha X$ is satisfied, for example, if each $p_{i}$ has a countable neighborhood base in $\alpha X$. Then each $\left\{p_{i}\right\}$ would be a closed $G_{\delta}$ set in the normal space $\alpha X$. By [6. p. 15], each $\left\{p_{i}\right\}$ would then be a zero set in $\alpha X$.

Lemma If $\alpha: X \rightarrow \alpha X$ is the inclusion map then $\mathcal{L}\{\alpha\}=\alpha X \backslash X$.

Proof. From the proof of Theorem 1 we see that $\pi_{\alpha}(\beta X \backslash X)=\mathscr{L}\{\alpha\}$ 
where $\pi_{\alpha}: \beta X \rightarrow \alpha X$ is the quotient map.

Corollary 2. Suppose $X=\cup{ }_{i=1}^{\infty} F_{i}$ where $F_{i}$ is compact, $F_{i+1} \supseteq F_{i}, X \backslash F_{i}$ is connected, $i=1,2, \ldots$, and each compact subset of $X$ is contained in some $F_{i}$. Then each remainder of $X$ is connected.

Proof. By the lemma $\alpha X \backslash X=\mathcal{L}\{\alpha\}=\bigcap_{i=1}^{\infty} \operatorname{cl} \alpha\left(X \backslash F_{i}\right)$ (the second equality is easily verified). This set is connected since it is the intersection of a decreasing sequence of compact connected subsets.

COROLlaRY 3. $\mathbf{R}^{n}$ has only connected remainders for $n>1$. Thus $\mathbf{R}^{n}$ has no remainders with cardinality $<c$ (except the one-point remainder) if $n>1$.

2. The nonlocally compact case. The remainder question for spaces which fail to have compact neighborhoods for some of their points is considerably more difficult and very little has been done. Rayburn [13] has obtained a result analogous to the one by Magill which was cited in the proof of Theorem 1. Using this, we are able to extend Theorem 1 to the nonlocally compact case. Denote by $R(X)$ the set of points of $X$ which have no compact neighborhoods. A continuous map $f: X \rightarrow Y$ is closed at $x \in X$ if for every neighborhod $U$ of $f^{-1}(f(x))$ there is a neighborhood of $V$ of $f(x)$ for which $f^{-1}(V) \subseteq U . f$ is perfect at $x$ if it is closed at $x$ and $f^{-1}(f(x))$ is compact. Note that $f$ is closed (perfect) if and only if it is closed (perfect) at each $x \in X$.

THeOREM 3. Let $f: X \rightarrow Y$ be continuous where $Y$ is compact. If $f$ is $1-1$ on $R(X)$ and perfect at each point of $R(X)$ then $\mathcal{L}\{f\} \backslash f(R(X))$ is a remainder of $X$.

Proof. The proof that $f^{\beta}(\beta X \backslash X)$ is dense in $\mathcal{L}\{f\}$ is exactly as in the proof of Theorem 1. Thus $f^{\beta}\left(\operatorname{cl}_{\beta X}(\beta X \backslash X)\right)=\mathcal{L}\{f\}$. In order to use Theorem 1.3 of [13] we need to show:

(i) $f^{\beta}(\beta X \backslash X) \subseteq \mathcal{L}\{f\} \backslash f(R(X))$.

(ii) $\left.f^{\beta}\right|_{R(X)}$ is a homeomorphism onto $f(R(X))$.

(iii) $f^{\beta}(R(X)) \cap f^{\beta}(\beta X \backslash X)=\varnothing$.

(This last condition, while not explicitly stated by Rayburn, is necessary for the validity of his theorem.) We would thus have a space $\mathcal{L}\{f\} \backslash f(R(X))$, a compactification $\mathcal{L}\{f\}$ of it, and a continuous map $f^{\beta}: \operatorname{cl}_{\beta X}(\beta X \backslash X) \rightarrow$ $\mathcal{L}\{f\}$ which carries $R(X)$ homeomorphically onto $\mathcal{L}\{f\} \backslash(\mathcal{L}\{f\} \backslash f(R(X)))$ $=f(R(X))$. (Note that $f(R(X)) \subseteq \mathcal{L}\{f\}$ since $R(X) \subseteq \operatorname{cl}_{\beta X}(\beta X \backslash X)$.)

(i) If $f^{\beta}(\beta X \backslash X)$ is not contained in $\mathcal{E}\{f\} \backslash f(R(X))$ then there are points $p \in \beta X \backslash X$ and $x \in R(X)$ with $f^{\beta}(p)=f(x)$. Since $f$ is perfect at $x$, $f^{-1}(f(x))$ is compact. Thus there are neighborhoods $U$ of $f^{-1}(f(x))$ and $V$ of $p$ in $\beta X$ with $U \cap V=\varnothing$. $U \cap X$ is open in $X$ so there is a neighborhood $W$ of $f(x)$ with $f^{-1}(W) \subseteq U \cap X$, since $f$ is closed at $x$. It follows that $f^{-1}(W) \cap V=\varnothing$. However, $p \in \operatorname{cl}_{\beta} X(V \cap X)$ so that $f^{\beta}(p) \in \operatorname{cl}_{Y}(f(V \cap$ $X)$ ). Now $W$ is a neighborhood of $f(x)=f^{\beta}(p)$ so that $W \cap f(V \cap X) \neq \varnothing$. 
We see that $f^{-1}(W) \cap V \neq \varnothing$, a contradiction.

(ii) By assumption, $\left.f^{\beta}\right|_{R(X)}$ is 1-1. The hypothesis that $f$ is closed at $x$ for each $x$ says that $\left.f\right|_{R(X)}=\left.f^{\beta}\right|_{R(X)}$ is a closed map. Thus $\left.f^{\beta}\right|_{R(X)}$ is a homeomorphism.

(iii) Immediate from (i).

Applying Rayburn's Theorem 1.3, we conclude that $\mathcal{L}\{f\} \backslash f(R(X))$ is a remainder of $X$.

We use Theorem 3 to establish a remainder theorem for a space $X$ for which $R(X)$ is a singleton which is contained in a compact subset with countable character. ( $F \subseteq X$ has countable character if there is a countable sequence of open sets $O_{1} \supseteq O_{2} \supseteq \cdots \supseteq F$ such that if $O$ is open in $X$ and $F \subseteq O$ then there is an $n$ for which $O_{n} \subseteq O$.)

LEMMA. If $R(X)=\{p\}$ and $p$ is contained in a compact subset $F$ having countable character then a subset of $(0,1]$ is a remainder of $X$.

Proof. For each $n$ there is a map $f_{n}: X \rightarrow[0,1]$ such that $f_{n}(F)=\{0\}$ and $f_{n}\left(X \backslash O_{n}\right)=\{1\}$. Let $f(x)=\Sigma 2^{-n} f_{n}(x)$. We have $f: X \rightarrow[0,1], f(F)=\{0\}$; and if $x \notin F$ we have $O_{n} \subseteq X \backslash\{x\}$ for some $n$ so that $f(x) \geqslant 2^{-n}$. It follows that $f^{-1}(f(p))=f^{-1}(0)=F$. We must show that $f$ is closed at $p$. Let $U$ be a neighborhood of $F$. There is an $n$ for which $O_{n} \subseteq U$. For $x \notin O_{n}$ we have $f(x) \geqslant 2^{-n}$. Thus, for the neighborhood $V=\left[0,2^{-n}\right)$ of $f(p)=0$ we have $f^{-1}(V) \subseteq U$. We conclude that $f$ is perfect at $p$. By Theorem 3 it follows that $\mathcal{L}\{f\} \backslash f(R(X)) \subseteq(0,1]$ is a remainder of $X$.

THEOREM 4. If $R(X)=\{p\}$ and $p$ is contained in a compact subset $F$ having countable character then there is a continuous $f: X \rightarrow[0,1]$, perfect at $p$, with $\mathcal{L}\{f\} \backslash f(R(X))$ equal to $(0,1]$ or to $\{1 / n \mid n=1,2, \ldots\}$.

Proof. By the lemma we have a $g: X \rightarrow[0,1]$ with $\mathcal{L}\{g\} \backslash g(R(X))=\alpha X$ $\backslash X \subseteq(0,1]$. If $(0, b] \subseteq \alpha X \backslash X$ for some $b>0$ then let $f=(g \wedge \bar{b}) / b$ where $\bar{b}: X \rightarrow\{b\}$. Then $f: X \rightarrow[0,1]$ and is perfect at $p . \mathcal{L}\{f\} \backslash f(R(X))=(0,1]$.

If there is no $b>0$ for which $(0, b] \subseteq \alpha X \backslash X$, then $[0,1] \backslash \mathcal{L}\{g\}$ is a union of open intervals and, since no $[0, b) \subseteq \mathcal{L}\{g\} \backslash g(R(X))=\mathcal{L}\{g\} \backslash\{0\}$, it follows that we can find two sequences $\left\{a_{n}\right\},\left\{b_{n}\right\}$ satisfying:

(i) $1=b_{1}>a_{1} \geqslant b_{2}>a_{2} \geqslant b_{3}>a_{3} \geqslant \ldots$

(ii) $a_{n} \rightarrow 0, b_{n} \rightarrow 0$.

(iii) $\left[b_{i+1}, a_{i}\right] \cap \mathcal{L}\{g\} \neq \varnothing$.

(iv) $\mathcal{L}\{g\} \backslash\{0\} \subseteq \cup\left[b_{i+1}, a_{i}\right]$.

(Note: 0 must be a limit point of $\mathcal{L}\{g\} \backslash g(R(X))$ since otherwise $\mathcal{L}\{g\} \backslash g(R(X))$ would be a compact remainder of $X$.)

Let $h:[0,1] \rightarrow[0,1]$ be defined as follows: The interval $\left[b_{i+1}, a_{i}\right]$ is mapped onto $1 /(i+1)$, the interval $\left[a_{i}, b_{i}\right]$ is mapped affinely onto the interval $[1 /(i+1), 1 / i], i=1,2, \ldots$, and $h(0)=0$. Let $f: X \rightarrow[0,1]$ be defined by $f=h \circ g$. Then $\mathcal{L}\{f\} \backslash f(R(X))=\{1 / n \mid n=1,2, \ldots\}$ and $f$ is perfect at $p$. 
LEMMA. $A$ closed, noncompact subset $A$ of a realcompact space $X$ contains $a$ $C$-embedded copy of $\mathbf{N}$.

Proof. Choose $p \in\left(\operatorname{cl}_{\beta X} A\right) \backslash A$. Then $p \in \beta X \backslash X$ and since $X$ is realcompact there is a continuous function $g: \beta X \rightarrow[0,1]$ with $g(p)=0$ and $g(x)>0$ for $x \in X[4$, Theorem 3.10.1]. Define $f: X \rightarrow \mathbf{R}$ by $f(x)=1 / g(x)$. Then $f$ is continuous and unbounded on $A$. By $[6,1.20] A$ contains a $C$-embedded copy of $\mathbf{N}$.

TheOREM 5. Suppose $X$ is realcompact, $R(X)=\{p\}$, and $p$ is contained in a compact set of countable character. Then $(0,1]$ is a remainder of $X$.

Proof. If not, then by Theorem 4 there is a continuous $f: X \rightarrow[0,1]$, perfect at $p$, such that $\mathcal{L}\{f\} \backslash f(R(X))=\{1 / n \mid n=1,2, \ldots\}$. Choose sequences $\left\{a_{n}\right\},\left\{b_{n}\right\}$ so that $a_{0}=b_{0}=1,1 /(n+1)<b_{n}<a_{n}<1 / n, n=$ $1,2, \ldots F_{n+1}=f^{-1}\left(\left[a_{n+1}, b_{n}\right]\right)$ is a closed, noncompact subset of $X$ (if it were compact, then $\left.1 /(n+1) \notin \operatorname{cl} f\left(X \backslash F_{n+1}\right)\right)$ so by the lemma $F_{n+1}$ contains a $C$-embedded copy of $\mathbf{N}$, say $\mathbf{N}_{n+1}$. Let $\pi:[0,1] \rightarrow[0,1]$ be defined in such a way that $\pi\left(\left[a_{n}, b_{n-1}\right]\right)=1 / n, \pi$ maps the interval $\left[b_{n}, a_{n}\right]$ affinely onto the interval $[1 /(n+1), 1 / n], n=1,2, \ldots$, and $\pi(0)=0$. If we let $\hat{f}=\pi \circ f$ then $\mathcal{L}\{\hat{f}\}-\hat{f}(R(X))=\{1 / n \mid n=1,2, \ldots\}$ and $\hat{f}^{-1}(1 / n)$ contains $\mathbf{N}_{n}$ for all $n$.

Let $g_{1}: X \rightarrow\left[a_{1}, 1\right]$ be a continuous function with $g_{1}\left(\mathbf{N}_{1}\right)=Q \cap\left(a_{1}, 1\right)$. Let $h_{1}: X \rightarrow[0,1]$ be such that $h_{1}\left(\hat{f}^{-1}\left(\left[0, b_{1}\right]\right)\right)=\{0\}, h_{1}\left(\hat{f}^{-1}\left(\left[a_{1}, 1\right]\right)\right)=\{1\}$. Let $t_{1}=g_{1} h_{1}$. Then $t_{1}(x) \in\left[a_{1}, 1\right]$ for all $x \in f^{-1}\left(\left[a_{1}, 1\right]\right)$ and $t_{1}\left(\mathbf{N}_{1}\right)$ is dense in $\left[a_{1}, 1\right]$. (Since $N_{1} \subseteq \hat{f}^{-1}\left(\left[a_{1}, 1\right]\right)$, we have $t_{1}\left(\mathbf{N}_{1}\right)=Q \cap\left(a_{1}, 1\right)$.) Also, $t_{1}(x)=$ 0 if $x \in \hat{f}^{-1}\left(\left[0, b_{1}\right]\right)$.

If we have defined $g_{n}$ and $h_{n}$ as continuous where $g_{n}: X \rightarrow\left[a_{n}, b_{n-1}\right]$ with $g_{n}\left(\mathbf{N}_{n}\right)=Q \cap\left(a_{n}, b_{n-1}\right)$ and $h_{n}: X \rightarrow[0,1]$ with $h_{n}\left(\hat{f}^{-1}\left(\left[a_{n}, b_{n-1}\right]\right)\right)=\{1\}$ and $h_{n}\left(\hat{f}^{-1}\left(\left[0, b_{n}\right]\right) \cup \hat{f}^{-1}\left(\left[a_{n-1}, 1\right]\right)\right)=\{0\}$, then let $t_{n}=g_{n} h_{n}$ and define

$$
t(x)=\left[\left(\sum t_{n}(x)\right) \wedge \underline{1}\right]+\hat{f}(x) .
$$

(There are no convergence problems since for any $x \in X$ there are at most two $t_{n}$ for which $t_{n}(x) \neq 0$ : For $x \notin \hat{f}^{-1}\left(\left[b_{n}, a_{n-1}\right]\right)$ we have $h_{n}(x)=0$ so $t_{n}(x)=0$.) Now $t^{-1}(t(p))=Z(t)$ since $t(p)=0$. This is compact since $Z(t)$ $=Z(\hat{f}) \cap\left(\cap Z\left(t_{n}\right)\right)$ and $Z(\hat{f}) \subseteq Z\left(t_{n}\right)$ for each $n$. It follows that $Z(t)=$ $Z(\hat{f})=\hat{f}^{-1}(f(p))$ is compact. To see that $t$ is closed at $p$ let $V$ be a neighborhood of $p$. Since $\hat{f}$ is perfect at $p$ there is a neigborhood $[0, \delta)$ of $\hat{f}(p)$ such that $\hat{f}^{-1}([0, \delta)) \subseteq V$. If $x \in t^{-1}([0, \delta))$ then $t(x) \in[0, \delta)$ so $\hat{f}(x)<\delta$. Thus $x \in V$. We see that $t^{-1}([0, \delta)) \subseteq V$. It follows that $t$ is perfect at $p$. Let $T=\mathcal{L}\{t\} \backslash t(R(X))$. Then $\left[a_{n+1}+1 /(n+1), b_{n}+1 /(n+1)\right] \subseteq T$ for all $n$. (Since any compact $F \subseteq X$ can contain only finitely many points of $N_{n+1}$.) Define $\theta:[0,2] \rightarrow[0,1]$ in such a way that $\theta\left(\left[b_{n-1}+1 / n, a_{n-1}+1 / n\right]\right)=$ $1 / n, \theta$ maps $\left[a_{n}+1 / n, b_{n-1}+1 / n\right]$ affinely onto $[1 /(n+1), 1 / n], n=$ $1,2,3, \ldots$, and $\theta(0)=0$. If we let $\phi=\theta \circ t$ we have $\phi(X \backslash F)$ is dense in $[0,1]$ for any compact $F \subseteq X$. (Clearly $F$ can contain at most finitely many 
points of $\cup \mathbf{N}_{n}$ and $\phi\left(\cup \mathbf{N}_{n}\right)$ is dense in $[0,1]$.) Thus $\mathcal{L}\{\phi\}=[0,1]$ and so $\mathcal{L}\{\phi\} \backslash \phi(R(X))=(0,1]$.

COROLlaRY. If $X$ is realcompact and $R(X)=\{p\}$ where $X$ has a countable neighborhood base at $p$, then $(0,1]$ is a remainder of $X$.

\section{REFERENCES}

1. P. S. Alexandroff and P. Urysohn, Mémoire sur les espaces topologiques compacts, Verh. Nederl. Akad. Wetensch. Afd. Natuurk. Sect. I 14 (1929), 1-96.

2. E. Cech, On bicompact spaces, Ann. of Math. (2) 38 (1937), 823-844.

3. R. E. Chandler, Hausdorff compactifications, Dekker, New York, 1976.

4. R. Engelking, Outline of general topology, PWN, Warsaw; Interscience, New York, 1968. MR 36 \#4508.

5. , On the double circumference of Alexandroff, Bull. Acad. Polon. Sci. Sér. Sci. Math. Astronom. Phys. 16 (1968), 629-634. MR 39 \#921.

6. L. Gillman and M. Jerison, Rings of continuous functions, Springer-Verlag, Berlin, 1977 (reprint). MR 22 \#6994.

7. K. D. Magill, Jr., N-point compactifications, Amer. Math. Monthly 72 (1965), 1075-1081. MR 32 \#3036.

8.

A note on compactifications, Math. Z. 9 (1966), 322-325. MR 34 \#3530.

9 , More on remainders of spaces in compactifications, Bull. Acad. Polon. Sci. Sér. Sci. Math. Astronom. Phys. 18 (1970), 449-451. MR 42 \#3751.

10. $\_$On the remainders of certain metric spaces, Trans. Amer. Math. Soc. 160 (1971), 411-417. MR 44 \#3289.

11. S. Mrówka, On the potency of subsets of $\beta \mathrm{N}$, Colloq. Math 7 (1959), 23-25. MR 22 \# 5018.

12. B. Pospisil, Remark on bicompact spaces, Ann. of Math. (2) 38 (1937), 845-846.

13. M. C. Rayburn, On Hausdorff compactifications, Pacific J. Math. 44 (1973), 707-714. MR 47 \# 5824.

14. J. W. Rogers, Jr., On compactifications with continua as remainders, Fund. Math. 70 (1971), 7-11. MR 44 \#993.

15. A. K. Steiner and E. F. Steiner, Compactifications as closures of graphs, Fund. Math. 63 (1968), 221-223. MR 38 \#6546.

Department of Mathematics, North Carolina State University, Raleigh, North Carolina 27607 